POLITICAS

\title{
El informe mundial sobre la violencia y la salud
}

\author{
Etienne G. Krug ${ }^{1}$, James A. Mercy ${ }^{2}$, Linda L. Dahlberg ${ }^{2}$, Anthony B. Zwi ${ }^{3}$ \\ ${ }^{1}$ Injuries and Violence Prevention Department, Non Communicable Diseases and Mental Health. WHO, \\ Geneva, Switzerland. \\ ${ }^{2}$ Division of Violence Prevention, National Center for Injury Prevention and Control, CDC, Atlanta, USA. \\ ${ }^{3}$ School of Public Health and Community Medicine, University of New South Wales, Sydney, Australia.
}

En 1966, la Organización Mundial de la Salud declaró la violencia como uno de los principales problemas de salud pública. Para dar seguimiento a dicha resolución, el 3 de octubre de este año, la OMS publicó el primer Informe mundial sobre la violencia y la salud, el cual analiza los diferentes tipos de violencia, incluidos el maltrato y abandono de menores, la violencia entre los jóvenes, la violencia contra la pareja, la violencia sexual, el maltrato a ancianos, la violencia autoinfligida y la violencia colectiva. Para todos estos tipos de violencia, el informe explora la magnitud de sus efectos en la salud y en la sociedad, los factores de riesgo y protección y los esfuerzos de prevención que se han desplegado. El lanzamiento del informe dará inicio a una Campaña Global de Prevención de la Violencia que durante un año se centrará en la aplicación de sus recomendaciones. Este artículo resume algunos de los puntos más importantes del informe mundial.

Palabras clave: violencia, salud pública, prevención.

\section{World report on violence and health}

In 1996, the World Health Assembly declared violence a major public health issue. To follow up on this resolution, on October 3 this year, WHO released the first World Report on Violence and Health. The report analyses different types of violence including child abuse and neglect, youth violence, intimate partner violence, sexual violence, elder abuse, self-directed violence, and collective violence. For all these types of violence, the report explores the magnitude of the health and social effects, the risk and protective factors, and the types of prevention efforts that have been initiated. The launch of the report will be followed by a 1-year Global Campaign on Violence Prevention, focusing on implementation of the recommendations. This article summarises some of the main points of the world report.

Key words: violence, public health, prevention.

Cerca de 4.400 personas mueren diariamente por actos autoinfligidos, interpersonales o colectivos de violencia. Miles más sufren lesiones u otras consecuencias no fatales sobre su salud como resultado de ser víctimas o testigos de hechos de violencia. Además, cientos de miles de vidas se ven destruidas y las familias disgregadas y son altísimos los costos en que se incurre para el

Traducido y reproducido con la autorización de los editores. Lancet 2002;360:1083-8

Correspondencia:

E.G. Krug, Injuries and Violence Prevention Department, WHO, Via Appia 20, 1211, Ginebra, Suiza

Recibido:17/10/02; aceptado: 24/11/02 tratamiento de las víctimas, el apoyo a las familias, la reparación de la infraestructura, la persecución de los perpetradores o como resultado de pérdidas en la productividad y la inversión.

La OMS ha disparado las alarmas al publicar recientemente el primer Informe mundial sobre la violencia y la salud (1) y lanzar una Campaña Global de Prevención de la Violencia. El informe analiza un amplio espectro de violencia, que incluye el maltrato y abandono de menores por parte de sus cuidadores, la violencia entre los jóvenes, la violencia contra la pareja, la violencia sexual, el maltrato a los ancianos, la violencia autoinfligida y la violencia colectiva. Para todos 
estos tipos de violencia, el Informe explora su magnitud y efecto en diferentes contextos culturales, sociales y económicos y describe los esfuerzos de prevención que se han iniciado. La OMS aspira a atraer mayor atención y recursos para la prevención de la violencia y para estimular la acción local, nacional e internacional.

\section{La historia de la violencia como problema de salud pública}

En muchos países, la prevención de la violencia es todavía un campo nuevo que apenas emerge en las acciones de salud pública. Sólo recientemente los actores de la salud pública han comenzado a tomar conciencia de las contribuciones que pueden hacer para reducir la violencia y mitigar sus consecuencias. En 1949, Gordon llamaba la atención sobre el hecho de que los esfuerzos de prevención de lesiones debían basarse en la comprensión de las causas, así como sucede con las enfermedades transmisibles y de otro tipo (2). En 1962, Abad Gómez, al referirse a la definición de salud dada por la OMS, señalaba que es obvio que la violencia no contribuye a "prolongar la vida" o a una "situación de cabal bienestar". Definía la violencia como un problema que los expertos en salud pública necesitaban abocar y que no debía ser de exclusivo dominio de los abogados, el personal militar o los políticos (3).

La atención prestada a la prevención de la violencia por parte de los expertos en salud pública ha aumentado sustancialmente desde la década de los 70; el número de publicaciones sobre violencia en Medline ha incrementado en un $550 \%$ (de 2.711 en los 70 a más de 8.000 en los 90 ). Durante ese mismo periodo, el número total de artículos aparecidos en Medline casi se dobló. Además de emprender la investigación científica, varios países desarrollaron otras actividades relacionadas con la violencia, fundamentalemente en el área de la recolección de información y de la atención a las víctimas. El número de organizaciones de la sociedad civil y de actividades dirigidas a responder a la violencia de género contra la mujer también incrementó de manera dramática. Los esfuerzos por incluir la violencia en la agenda global de salud pública culminaron en 1996 con la adopción de una resolución por parte de la Asamblea Mundial de la Salud, el foro anual de todos los ministros de salud (4). Dicha resolución declaraba la violencia como uno de los problemas de salud pública más acuciantes y hacía un llamado a incrementar las acciones.

\section{¿Por qué el sector de salud pública debe estar involucrado en la prevención de la violencia?}

El sector de la salud pública tiene injerencia directa en el problema de violencia no sólo por sus gigantescos efectos sobre la salud y los servicios de atención, sino también por las contribuciones significativas que puede y debe aportar para reducir sus consecuencias. Los trabajadores de la salud pública pueden hacer aportes significativos en esta área a través de su enfoque preventivo y científico, así como de su potencial para coordinar esfuerzos multidisciplinarios y multisectoriales y de su papel en asegurar la disponibilidad de los servicios de atención a las víctimas.

La salud pública complementa los enfoques existentes para enfrentar la violencia, los cuales son principalmente reactivos, al centrar su atención en la transformación de los factores sociales, de comportamiento y de medio ambiente que dan origen a la violencia (5). Tal visión tiene sus raíces en las tradiciones y conceptos de salud pública que se han aplicado con éxito en la reducción de otros problemas de salud pública como la viruela, las lesiones por accidentes de tráfico y la poliomielitis. Existe cada vez más evidencia y mayor compromiso con la idea de que la prevención de la violencia sí funciona. El sector de la salud pública también se apoya de manera primordial en la utilización de la evidencia científica en el diseño de políticas. Si queremos tener éxito en la prevención de la violencia, las políticas y programas que diseñemos deben apoyarse en la ciencia, así como en otras estrategias exitosas de salud pública.

Las actividades de prevención de la violencia involucran de manera particular asociaciones con diversos sectores de la sociedad, campos científicos y organizaciones. El papel central de 
las comunidades en la prevención de la violencia también se ha convertido en tópico común y la salud pública tiene un compromiso de vieja data con el apoyo y la ayuda a las comunidades en la resolución de sus propios problemas de salud. De igual manera, juega un papel importante al asegurar la disponibilidad de los servicios de atención necesarios (6). Dicho papel puede extenderse a los servicios de salud a fin de reducir la gravedad y duración de las lesiones y efectos incapacitantes, tanto físicos como psicológicos, de las personas afectadas por incidentes violentos. Es evidente, por ejemplo, que los sistemas de respuesta a emergencias y traumas son componentes críticos de los servicios de salud en una aproximación integral a la prevención y manejo de la violencia.

\section{El informe y lo que espera lograr}

\section{Generalidades}

El informe es el resultado de tres años de trabajo de más de 160 expertos en más de 70 países y de reuniones regionales de consulta realizadas en Africa, las Américas, Asia, Europa y el Medio Oriente. Mucha de la información referente a violencia y salud en el ámbito global está ahora compilada en un solo documento. Es la primera vez que la OMS asume una posición tan clara y visible a favor de la prevención de la violencia. Con la adhesión de líderes políticos mundiales como Nelson Mandela, Kofi Annan y Oscar Arias, el informe será una herramienta poderosa para movilizar a quienes toman las decisiones en todo el mundo. La directora general de la OMS ha comprometido a la organización para que juegue un papel importante en la prevención de la violencia: "La salud pública no ofrece todas las respuestas a este problema complejo de la violencia, pero estamos decididos a desempeñar nuestro papel en su prevención a escala mundial. Este informe contribuirá a estructurar la respuesta del mundo a la violencia y a hacer de éste un lugar más seguro y saludable para todos".

\section{Definición y tipos de violencia}

El informe acoge la definición de violencia redactada por un grupo de trabajo de la OMS en 1996: "El uso deliberado de la fuerza física o el poder, ya sea en grado de amenaza o efectivo, contra uno mismo, otra persona o un grupo o comunidad, que cause o tenga muchas probabilidades de causar lesiones, muerte, daños psicológicos, trastornos del desarrollo o privaciones" (7). Esta deficinión comprende todos los tipos de violencia y cubre una amplia gama de actos, tanto por comisión como por omisión, que van más allá de los resultados letales y de las lesiones provenientes de la violencia.

En el informe, la violencia se presenta en tres grandes categorías: autoinfligida, interpersonal y colectiva (figura 1). Cada categoría se subdivide con el fin de incluir los tipos específicos de violencia, los contextos de la violencia y la naturaleza de los actos violentos (física, sexual, psicológica, así como la deprivación o desatención). Aunque el análisis de los tipos específicos de violencia es valioso, también es importante entender las relaciones entre ellos. Por ejemplo, las víctimas de abuso a menores tienen una probabilidad por encima del promedio de desplegar comportamientos agresivos y violentos al llegar a la adolescencia y la edad adulta (8-10) y el abuso sexual durante la infancia o la adolescencia ha sido asociado con el comportamiento suicida (11). Muchos factores de riesgo, tales como el abuso del alcohol, el acceso a armas de fuego o las desigualdades económicas, también son comunes en la mayoría de los tipos de violencia. Estas asociaciones son importantes porque muestran el potencial de la prevención en varios tipos de violencia a través de intervenciones orientadas a unos pocos factores de riesgo. De igual manera, sustentan la necesidad de una mayor colaboración entre los grupos dedicados a la prevención de diversas formas de violencia.

\section{Magnitud}

Los datos a escala mundial y nacional son escasos. Sin embargo, se estima que 1,6 millones de personas murieron en forma violenta en el 2000 (12), es decir 28,8 por 100.000 habitantes. Casi la mitad de estas muertes fueron suicidios, cerca de la tercera parte fueron homicidios y una quinta parte por eventos relacionados con conflictos armados. Las cifras varían considerablemente entre y dentro de los países. 


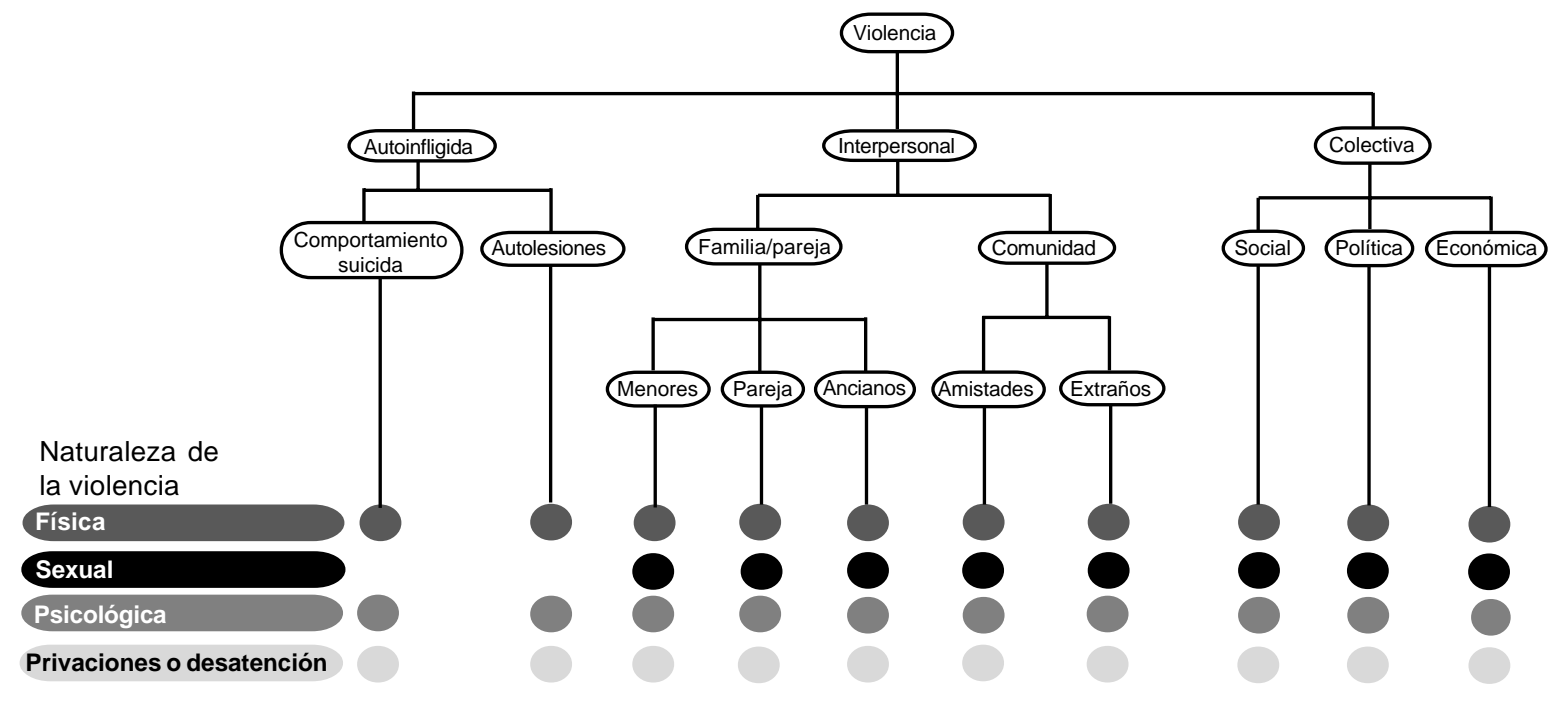

Figura 1. Clasificación de la violencia.

Por ausencia de información confiable es difícil llegar a estimativos globales para los diferentes tipos de abuso. No obstante, la naturaleza generalizada de la violencia es evidente: en 48 estudios de población en todo el mundo, entre el 10 y el $69 \%$ de las mujeres informaron haber sido agredidas físicamente por su pareja a lo largo de su vida (13); cerca del $20 \%$ de las mujeres y entre 5 y $10 \%$ de los hombres dijeron haber sido sexualmente abusados en su infancia $(14,15)$, y los resultados de los pocos estudios realizados entre personas mayores muestran que entre el 4 y el $6 \%$ son maltratadas en su hogares (16-20).

Muchas investigaciones han demostrado que las consecuencias de la violencia sobre la salud exceden en mucho las cifras de muertes y lesiones. Las víctimas de la violencia tienen riesgos de sufrir problemas psicológicos y de comportamiento, incluida la depresión, el abuso del alcohol, la ansiedad y el comportamiento suicida, así como problemas en la salud reproductiva tales como las enfermedades de transmisión sexual, el embarazo no deseado y la disfunción sexual $(13,21-23)$.

\section{Causas}

La violencia no puede atribuirse a una sola causa, pues éstas son complejas y ocurren en diferentes niveles. Para representar dicha complejidad, el informe recurre a un modelo ecológico con cuatro niveles (24-30). El primero identifica factores biológicos y personales que influyen en la forma en que los individuos se comportan y que aumentan sus probabilidades de convertirse en víctimas o perpetradores de violencia, como son las características demográficas (edad, educación, ingreso), los desórdenes de la personalidad, el abuso de sustancias y el tener antecedentes como testigos o actores de comportamiento violento.

El segundo nivel se centra en las relaciones interpersonales cercanas, como las que se tienen con la familia y los amigos. En la violencia entre jóvenes, por ejemplo, tener amigos que cometen o provocan actos violentos puede incrementar el riesgo de ser víctima o perpetrador de violencia $(31,32)$. En cuanto a la violencia contra la pareja, el marcador más consistente en este nivel del modelo es el conflicto marital o la discordia en la relación. En la violencia contra ancianos, factores importantes son el estrés debido a la naturaleza pasada de la relación entre la persona abusada y el cuidador, así como las condiciones de hacinamiento en el lugar de vivienda.

El tercer nivel explora el contexto comunitario, o sea, escuelas, colegios, lugares de trabajo y barrios. El riesgo en este nivel se ve afectado por factores tales como la existencia de tráfico local 
de drogas, la ausencia de redes sociales y la pobreza. Se ha demostrado que todos éstos son importantes en varios tipos de violencia.

Finalmente, el cuarto nivel está enfocado hacia el amplio rango de factores sociales que contribuyen a crear un clima que bien propicia o inhibe la violencia: la capacidad de respuesta del sistema de justicia, las normas sociales y culturales relativas a los papeles que asumen los géneros 0 que rigen las relaciones padres-hijos, el desequilibrio en el ingreso, la fortaleza del sistema de seguridad social, la aceptación social de la violencia, el acceso a armas de fuego, la exposición a la violencia en los medios de comunicación y la inestabilidad política.

\section{Lecciones aprendidas sobre la prevención}

El modelo ecológico también puede utilizarse como marco para la prevención de la violencia, pues lograrlo implica actuar transversalmente en diferentes niveles simultáneamente. Los programas que se centran en el individuo tienden a promover actitudes y comportamientos positivos en los menores y jóvenes y pueden cambiar el comportamiento de individuos ya inmersos en las prácticas violentas. Los enfoques relacionales se usan para influir las interacciones intrafamiliares y las influencias negativas de los compañeros. Los esfuerzos orientados hacia la comunidad pueden estimular la acción comunitaria u orientarse hacia el cuidado y el apoyo a las víctimas. Por último, los enfoques sociales se dirigen a las condiciones económicas, las normas culturales y a ámbitos de gran impacto social como los medios masivos de comunicación.

De nuestra comprensión de la naturaleza y de la prevención aplicable a los tipos de violencia fundamentales surgieron algunas estrategias comunes. Primero, la familia desempeña un papel fundamental al influir directamente en la propensión al comportamiento violento, ya que puede ejercer influencias tanto protectoras como inductoras de riesgo sobre la probabilidad de que los hijos se involucren en actos de violencia interpersonal y en comportamientos suicidas a medida que crecen. La creación de ambientes que enfaticen la capacidad de la familia de proteger a los hijos de la violencia debe ser una actividad clave. Otra línea de acción promisoria se orienta hacia esfuerzos para proveer a los padres con información que puedan utilizar para criar a sus hijos de manera más eficiente en ambientes difíciles.

La exposición de los menores a la violencia en su forma de maltrato físico, sexual o emocional, de desatención o aún de situaciones que los convierten en testigos de actos violentos en el hogar tiene efectos significativos sobre el bienestar de los niños. Tal exposición constituye un factor de riesgo para una serie de comportamientos y desórdenes (por ejemplo, el consumo de cigarrillos, la obesidad, los comportamientos sexuales riesgosos y la depresión) que, a su turno, están causalmente relacionados con otros graves problemas de salud pública como el cáncer, las enfermedades cardiacas, las enfermedades de transmisión sexual y los suicidios (33). Las intervenciones tempranas para reducir la exposición infantil a la violencia son factores claves en la reducción de consecuencias de la violencia que tienen mucho alcance y que se expresan en los menores a medida que crecen para convertirse en adolescentes y adultos.

Las normas y valores sociales pueden jugar un papel de primer orden en la justificación de la victimización de mujeres y niños. El comportamiento suicida y aún la guerra también se justifican más fácilmente en algunos entornos culturales. Debe prestarse mucha atención a las diversas tradiciones sociales y culturales que coexisten en el mundo. La noción de que la violencia contra la mujer, los menores u otros seres humanos puede justificarse debe reconsiderarse a la luz del enorme costo social y en salud que la violencia impone en sus víctimas y en las sociedades. La promoción de normas y valores que muestren la violencia como algo ilegítimo e irresponsable pueden ser de gran importancia en la generación de contextos sociales intolerantes a la violencia y que brinden consideración a sus víctimas.

Las condiciones económicas son a la vez causa y efecto de la violencia. Casi en todas las sociedades, los pobres sobrellevan de manera desproporcionada la carga de salud pública 
generada por la violencia. El desequilibrio en los ingresos, en particular, se asocia con las tasas nacionales de homicidio (34). La violencia interpersonal y la guerra impiden el desarrollo económico al incrementar los costos de la salud y la inversión en seguridad, reduciendo así la productividad y el valor de la propiedad, traumatizando la prestación de servicios y socavando la gobernabilidad. La amenaza de la violencia puede desestabilizar las economías nacionales y regionales al comprometer el establecimiento y la viabilidad de empresas productivas. En consecuencia, no podemos separar las políticas y los programas económicos de la prevención de la violencia. Los enfoques integrales en la prevención de la violencia deben incluir esfuerzos encaminados a promover el desarrollo económico positivo, especialmente orientada a buscar la reducción de las desigualdades.

El informe de la OMS muestra que las intervenciones en la primera infancia, como las visitas a los hogares, reducen el maltrato y se cuentan entre las estrategias a largo plazo más prometedoras para reducir la violencia entre los jóvenes. Los programas de terapia grupal para padres y familias también tienen efectos duraderos y positivos sobre el comportamiento violento y delincuencial, además de ser costo-efectivas. Los programas orientados a desarrollar habilidades para la vida y competencias sociales son aproximaciones prometedoras para abocar el problema de la violencia interpersonal y los tratamientos de enfermedades mentales y los programas de terapia del comportamiento pueden reducir los comportamientos suicidas. Otras medidas como la reducción del acceso a instrumentos propios del suicidio y el homicidio han reducido las tasas de tales incidentes en algunos contextos. El informe muestra, igualmente, que pocos programas han sido sometidos a una evaluación rigurosa y que también existe un desequilibrio en cuanto al enfoque, ya que las estrategias comunitarias y sociales no han sido suficientemente enfatizadas en comparación con los programas orientados a los factores individuales y de relaciones interpersonales.

\section{Vías de acción propuestas}

Se requiere desarrollar planes nacionales de acción en colaboración con todas las agencias implicadas para asegurar que las organizaciones gubernamentales y no gubernamentales acuerden prioridades y objetivos, definan las responsabilidades de cada cual y trabajen mancomunadamente en el logro de estos objetivos. Los planes deben incluir la revisión y reforma de la legislación y las políticas, la generación de capacidades para la recolección de información y la investigación, el fortalecimiento de la atención a las víctimas y el desarrollo y evaluación de respuestas preventivas. Para asegurar que el plan trascienda las palabras hacia la acción, una organización específica debe ser autorizada para monitorizar e informar periódicamente sobre los avances.

Nuestra comprensión de la magnitud y las causas de la violencia debe mejorar. Los datos sobre los costos humanos, sociales y financieros de la violencia son importantes para entender el tema, establecer prioridades y convocar mayores esfuerzos preventivos. Sin embargo, la cantidad y la calidad de la información son pobres en todo el mundo. Más aún, a menudo los datos no son comparables entre países y regiones debido a las diferencias en la definición, los métodos de recolección de datos y los sistemas de clasificación. Se requiere de mayores esfuerzos para recolectar la información en forma estandarizada y asegurar su amplia difusión. Aunque se han logrado progresos en la comprensión de los factores que causan la violencia y aquéllos que contribuyen a su prevención, se necesita mucha más investigación, especialmente de factores sociales y culturales más amplios, incluidos los relacionados con la globalización.

Una de las áreas prioritarias sobre las que llama la atención el informe es la inversión en la prevención primaria: intervenciones tempranas para prevenir que los niños se conviertan en perpetradores de la violencia. Varias intervenciones de prevención primaria son prometedoras. Los esfuerzos de prevención deben integrarse a las políticas sociales y educativas 
para así reducir las desigualdades por razones de género o sociales, las cuales constituyen factores de riesgo muy importantes en la mayoría de los tipos de violencia. Las desigualdades pueden combatirse sólo a través de un conjunto de intervenciones que incluyen las reformas legales, el fortalecimiento de los servicios de protección social, la educación y la intercesión.

Uno de los ámbitos en los cuales el sector de la salud pública tiene una especial responsabilidad es en asegurar la disponibilidad de los servicios de atención a las víctimas de la violencia. Los servicios de emergencia y la atención a más largo plazo se deben mejorar para que provean una respuesta integral a las víctimas. Tabúes muy difundidos a menudo impiden reconocer y atender adecuadamente a las víctimas de la violencia sexual, de la autoinfligida, del maltrato a menores y de la ejercida contra mujeres y ancianos. Más aún, se deben desplegar esfuerzos para dar respuestas que integren los servicios médicos, legales y sociales requeridos.

Muchas agencias vienen trabajando para enfrentar el problema nacional e internacionalmente. Sin embargo, existen pocos mecanismos para promover la colaboración entre agencias y especialidades. La OMS realizó una reunión para incrementar la colaboración entre las agencias de las Naciones Unidas, la cual desembocó en la publicación de La guía de las Naciones Unidas sobre los recursos y las actividades de prevención de la violencia interpersonal (35). En las Américas, la Coalición Interamericana de Prevención de la Violencia se apoya en las fortalezas de seis agencias para desarrollar prevención multisectorial de la violencia, ellas son: los Centros para la Prevención y el Control de Enfermedades (CDC), el Banco Interamericano de Desarrollo, la Organización de Estados Americanos, la Organización Panamericana de la Salud, la Organización de Naciones Unidas para la Educación, la Ciencia y la Cultura y el Banco Mundial. Los objetivos fundamentales de dicha coalición son elevar la conciencia entre quienes toman decisiones, los formadores de opinión y los dirigentes cívicos en torno a los costos sociales y económicos de la violencia, así como sobre la necesidad de trascender los enfoques tradicionales de lucha contra el crimen basados en el control para promover los que se orientan hacia la prevención y establecer procedimientos de coordinación entre organismos multilaterales para subrayar el éxito de las intervenciones a escala nacional y local.

La adhesión a los tratados internacionales y otros mecanismos de protección de los derechos humanos debe incentivarse y fortalecerse. Varios instrumentos internacionales de relevancia directa en la prevención de la violencia se han firmado en las décadas pasadas. Los países podrían intensificar sus esfuerzos para ratificar dichos instrumentos y adaptar su legislación nacional a sus disposiciones. Por último, se deben buscar respuestas prácticas consensuadas internacionalmente contra el tráfico mundial de drogas y de armas, pues dada la magnitud de su efecto global, aún los éxitos más pequeños en esta área pueden cambiar la vida de muchas personas.

\section{Los retos del movimiento de prevención de la violencia}

Son muchos los retos que enfrenta este movimiento de prevención de la violencia. El primero y más importante reside en que debemos convencer a los diseñadores de las políticas, a los ministros de salud y a la opinión general de que los programas y las estrategias de prevención pueden ser costo-efectivas comparadas con alternativas tales como el encarcelamiento. Aunque hace falta mayor evidencia científica en muchos campos, existe la suficiente en los estudios sobre algunos tipos de violencia. Por ejemplo, en un análisis económico de varias estrategias de prevención de la violencia comparadas con medidas de encarcelamiento realizado en California se concluyó que los incentivos para la terminación del bachillerato y la capacitación a los padres de familia eran más costo-efectivos que el enfoque de sentencia mínima de prisión para ofensores reincidentes (36).

Otro gran desafío es el de convencer a las instituciones de salud pública nacionales y locales de que la prevención de la violencia es parte legítima e importante de su misión. Los ministros de salud pueden ser actores y facilitadores de primera línea en la prevención. Actualmente, la 
prevención de la violencia no se considera, en general, como problema prioritario de salud pública y menos aún como actividad legítima de salud pública en la mayoría de los ministerios de salud. Sin embargo, dicha actitud está cambiando. Por ejemplo, el ministerio de salud de Mozambique ha apoyado la elaboración de un plan nacional de prevención de la violencia y las facultades de salud pública ofrecen cada vez más cursos en epidemiología de la violencia e integran cada vez más los temas de prevención de la violencia en sus currículos.

Otro desafío significativo es la generación de un sentido de apropiación del problema y de responsabilidad para abocar el problema en el ámbito de las comunidades. El empoderamiento de las comunidades es esencial porque muchas de las soluciones más importantes tendrán que desarrollarse en el ámbito local. El valor de ese sentido de apropiación y de un compromiso sostenido con la prevención de la violencia se ha visto de manera dramática en Bogotá, Colombia, en donde tres alcaldías sucesivas han apoyado y dado continuidad a la aplicación de políticas y programas multifacéticos de prevención de la violencia que incluyen la restricción a la venta de alcohol y al porte de armas durante fines de semana y fechas especiales, la movilización de la comunidad y el desarrollo de infraestructura. Las tasas de homicidio en Bogotá han disminuido sustancialmente y tal disminución se atribuye, en parte, a la prohibición intermitente al porte armas en toda la ciudad (37).

El liderazgo mundial será necesario para estimular y proveer asistencia técnica a los esfuerzos de prevención de la violencia en todo el planeta. Sin la creación de organismos internacionales con capacidad para ejercer tal liderazgo, el desarrollo y aplicación de políticas y programas efectivos de prevención de la violencia avanzará con lentitud y dificultad. Ese liderazgo puede mejorar también la capacidad de las naciones para aprender unas de otras.

Si queremos prevenir la violencia debemos ser capaces de medirla y vigilarla. El desarrollo de sistemas de vigilancia que recojan información básica sobre la magnitud y el carácter de las lesiones y las muertes causadas por la violencia de manera sistemática y continua es un reto en todos los rincones del mundo. Afortunadamente, son muchos los esfuerzos que ya están en marcha para desarrollar tales sistemas. Por ejemplo, a comienzos de este año, la OMS y el CDC publicaron las Guías para la vigilancia de lesiones. De igual manera, se han venido elaborando los estándares para la clasificación de la información sobre lesiones: la clasificación internacional de las causas externas de lesiones tiene como fin ayudar a los investigadores y profesionales de la prevención a entender de manera más precisa las características de las lesiones que estudian, responder a interrogantes sobre las circunstancias en que se produjeron y facilitar información más detallada sobre categorías específicas de lesiones tales como las producidas por asaltos e intentos suicidas. Dichos estándares han sido utilizados para desarrollar un departamento de urgencias en el marco del sistema de vigilancia de lesiones en Jamaica (38). La información generada por este sistema se utiliza para orientar las actividades de prevención de la violencia.

\section{Conclusión}

Con la publicación del Informe mundial sobre la violencia y la salud, la comunidad internacional cuenta ahora con una compilación de parte del mejor conocimiento disponible sobre la prevención de la violencia y el papel del sector de la salud pública. El informe deberá servir de catalizador para el debate y la acción. Durante la campaña de un año que se inició con su lanzamiento, la OMS y sus socios llevarán este debate a varios países del mundo para promover su utilización generalizada y la cristalización de sus recomendaciones en políticas y acciones.

\section{Nota sobre conflicto de intereses}

Los autores de este artículo coeditaron el informe de la OMS.

\section{Referencias}

1. Krug EG, Dahlberg LL, Mercy JA, Zwi AB, Lozano $\mathbf{R}$, editors. World report on violence and health. Geneva: World Health Organization; 2002.

2. Gordon JE. The epidemiology of accidents. Am J Public Health 1949;504-15. 
3. Abad Gomez H. Violence requires epidemiological studies. Tribuna Médica 1962;2:1-12.

4. World Health Assembly. Prevention of violence: public health priority (WHA 49,25). Geneva: World Health Organization; 1996.

5. Mercy JA, Rosenberg ML, Powell KE, Broome CV, Roper WL. Public health policy for preventing violence. Health Aff (Millwood) 1993;12:7-29.

6. Committee for the Study of the Future of Public Health, Division of Health Care Services, Institute of Medicine. The future of public health. Washington, D.C.: National Academy Press; 1988.

7. World Health Organization. Global consultation on violence and health. Violence: a public health priority (WHO/EHA/SPI.POA.2). Geneva: World Health Organisation; 1996.

8. Farrington DP. The family backgrounds of aggressive youths. En: Hersov LA, Berger M, Shaffer D, editors. Aggression and antisocial behavior in childhood and adolescence. Oxford: Pergamon Press; 1978. p.73-93.

9. McCord J. A forty year perspective on the effects of child abuse and neglect. Child Abuse Negl 1983;7:26570.

10. Widom CS. Child abuse, neglect, and violent criminal behavior. Criminology 1989;27:251-72.

11. Paolucci EO, Genuis ML, Violato C. A meta-analysis of the published researchon the effects of child sexual abuse. J Psychol 2001;135:17-36.

12. Krug EG, Dahlberg LL, Mercy JA, Zwi AB, Lozano $\mathbf{R}$, eds. Statistical annex: world report on violence and health. Geneva: World Health Organization; 2002.

13. Heise LL, Ellsberg M, Gottemoeller M. Ending violence against women (population reports, series $L$, no 11). Baltimore: Johns Hopkins University School of Public Health, Center for Communications Programs; 1999.

14. Finkelhor D. The international epidemiology of child sexual abuse. Child Abuse Negl 1994;18:409-17.

15. Finkelhor D. Current information on the scope and nature of child sexual abuse. Future Child 1994;4:3153.

16. Pillemer K, Finkelhor D. Prevalence of elder abuse: a random sample survey. Gerontologist 1988;28:51-7.

17. Podnieks E. National survey on abuse of the elderly in Canada. J Elder Abuse Negl 1992;4: 5-58.

18. Kivelä SL, et al. Abuse in old age: epidemiological data from Finland. J Elder Abuse Negl 1992;4:1-18.

19. Ogg J, Bennett GCJ. Elder abuse in Britain. BMJ 1992; 305:998-9.

20. Comijs HC, Pot AM, Smit JH, Bouter LM, Jonker C. Elder abuse inthe community: prevalence and consequences. J Am Geriatr Soc 1998;46:885-8.
21. Fergusson DM, Horwood MT, Lynskey LJ. Childhood sexual abuse and psychiatric disorder in young adulthood: II psychiatric outcomes of childhood sexual abuse. J Am Acad Child Adolesc Psychiatry 1996;35:1365-74.

22. Davidson JR, Hughes DC, George LK, Blazer DG. The association of sexual assault and attempted suicide within the community. Arch Gen Psychiatry 1996;53: 550-5.

23. Wiederman MW, Sansone RA, Sansone LA. History of trauma and attempted suicide among women in a primary care setting. Violence Vict 1998;13:3-9.

24. Garbarino J, Crouter A. Defining the community context for parentchild relations: the correlates of child maltreatment. Child Dev 1978;49:604-16.

25. Bronfenbrenner V. The ecology of human development: experiments by nature and design. Cambridge: Harvard University Press; 1979.

26. Garbarino J. Adolescent development: an ecological perspective. Columbus: Charles E Merrill; 1985.

27. Tolan P, Guerra N. What works in reducing adolescent violence: an empirical review of the field. Boulder: University of Colorado, Center for the Study and Prevention of Violence; 1994.

28. Heise LL. Violence against women: an integrated ecological framework. Violence Against Women 1998;4: 262-90.

29. Schiamberg LB, Gans D. An ecological framework for contextual risk factors in elder abuse by adult children. J Elder Abuse Negl 1999;11:79-103.

30. Carp RM. Elder abuse in the family: an interdisciplinary model for research. New York: Springer; 2000.

31. Thornberry TP, Huizina D, Loeber R. The prevention of serious delinquency and violence: implications from the program of research on the causes and correlates of delinquency. In: Howell JC, Krisberg B, Hawkins JD, Wilson JJ, editors. Sourcebook on serious, violent and chronic juvenile offenders. Thousand Oaks: Sage; 1995. p.213-37.

32. Lipsey MW, Derzon JH. Predictors of serious delinquency in adolescence and early adulthood: a synthesis of longitudinal research. In: Loeber R, Farrington DP, editors. Serious and violent juvenile offenders: risk factors and successful interventions. Thousand Oaks: Sage; 1998. p.86-105.

33. Felitti VJ, Anda RF, Nordenberg D, et al. Relationship of childhood abuse and household dysfunction to many of the leading causes of death in adults: The Adverse Childhood Experiences (ACE) study. Am J Prev Med 1998;14:245-58.

34. Fajnzylber P, Lederman D, Loayza N. Inequality and violent crime. Washington, D.C.: Regional Studies Program, Office of the Chief Economist for Latin America and the Caribbean, World Bank; 1999. 
35. World Health Organization. Guide to United Nations resources and activities for the prevention of interpersonal violence (WHO/NMH/VIP/02.05). Geneva: World Health Organization; 2002.

36. Greenwood PW, Model KE, Rydell CP, Chiesa J. Diverting children from a life of crime: measuring costs and benefits. Santa Monica: Rand; 1996.

37. Villaveces A, Cummings $P$, Espitia VE, Koepsell TD, McKnight B, Kellermann AL. Effect of a ban on carrying firearms on homicide rates in two Colombian cities. JAMA 2000;283:1205-09.

38. Ward E, Durant T, Thompson M, Gordon G, Mitchell W, Ashley $D$, and the VRISS working group. Implementing a hospitalbased violence-related injury surveillance system-a background to the Jamaican experience. J Injury Control Safety Promotion (in press).

Traducción: Martha Renza 